\title{
New Approach for Finding Basic Performance Measures of Single Server Queue
}

\author{
Siew Khew Koh, ${ }^{1}$ Ah Hin Pooi, ${ }^{2}$ and Yi Fei Tan ${ }^{3}$ \\ ${ }^{1}$ Faculty of Engineering and Sciences, Universiti Tunku Abdul Rahman, Jalan Genting Kelang, 53300 Setapak, \\ Wilayah Persekutuan, Malaysia \\ ${ }^{2}$ Sunway University Business School, Sunway University, 47500 Subang Jaya, Selangor, Malaysia \\ ${ }^{3}$ Faculty of Engineering, Multimedia University, 63100 Cyberjaya, Selangor, Malaysia \\ Correspondence should be addressed to Siew Khew Koh; kohsk@utar.edu.my
}

Received 30 June 2014; Revised 22 September 2014; Accepted 22 September 2014; Published 20 October 2014

Academic Editor: Steve Su

Copyright (c) 2014 Siew Khew Koh et al. This is an open access article distributed under the Creative Commons Attribution License, which permits unrestricted use, distribution, and reproduction in any medium, provided the original work is properly cited.

Consider the single server queue in which the system capacity is infinite and the customers are served on a first come, first served basis. Suppose the probability density function $f(t)$ and the cumulative distribution function $F(t)$ of the interarrival time are such that the rate $f(t) /[1-F(t)]$ tends to a constant as $t \rightarrow \infty$, and the rate computed from the distribution of the service time tends to another constant. When the queue is in a stationary state, we derive a set of equations for the probabilities of the queue length and the states of the arrival and service processes. Solving the equations, we obtain approximate results for the stationary probabilities which can be used to obtain the stationary queue length distribution and waiting time distribution of a customer who arrives when the queue is in the stationary state.

\section{Introduction}

The GI/G/1 queue has been given a lot of attention due to its applications to a variety of manufacturing systems, communication systems, computer systems, and other related areas. The steady-state waiting time distribution and queue length distribution are important measures of performance in this model.

When the interarrival and service times are discrete random variables, the GI/G/1 queue is referred to as a discretetime queue. Several authors determined the steady-state waiting time distribution in the discrete-time GI/G/1 queue by using the Wiener-Hopf factorization (see [1-5]), the polynomial factorization (see $[6,7]$ ), and the matrix-analytic method (see $[8,9])$.

Steady-state waiting time distribution in the continuoustime GI/G/1 queues can be found by numerical approximations based on the theory of Fredholm integral equations [10].

In Kim and Chaudhry [11], a discrete-time version of the distributional Little's law is established. Based on this law, the queue length distribution for the discrete-time GI/G/1 queue may be obtained from its waiting-time distribution.

Neuts [12] approximated the general distributions of service time and arrival time with phase-type distributions and solved the steady-state distribution using the matrixgeometric approach.

In this paper, we consider a single server queue in which the service time and interarrival time are assumed to have constant asymptotic rates. Abbreviating "constant asymptotic rate" as "CAR," we may denote the resulting queue as a CAR/ $\mathrm{CAR} / 1$ queue and any distribution with a constant asymptotic rate as a CAR distribution. In practice many distributions such as exponential, Erlang, hyperexponential, and gamma satisfy this requirement. However the heavy-tailed arrival time distributions which are often used in queuing analysis are not CAR. In order to be still able to apply the results in this paper, we may attempt to approximate the non-CAR distributions by CAR distributions. For example, in the case when there is a value $T^{*}$ such that the probability of the given random variable $Y$ larger than $T^{*}$ is negligible, we may 
approximate the distribution of $Y$ by a CAR distribution by changing the rate of the distribution of $Y$ at time $t$ for all $t>T^{*}$ to be all equal to the rate at time $T^{*}$.

To analyze the CAR/CAR/1 queue, we may first discretize it by segmenting the time axis into a sequence of equal intervals of length $\Delta t$. We next derive a set of equations for the stationary probabilities of the queue length and the states of the arrival and service processes. By solving the equations, we obtain approximately the stationary probabilities. Each of the probabilities may be found more accurately by an extrapolation of the probability on the values of $\Delta t$.

From the stationary queue length distribution, we have also obtained the waiting time distribution and sojourn time distribution of a customer who arrives when the queue is in a stationary state.

The approach which is closely related to the methods in this paper is the polynomial factorization used in [7]. Instead of taking note of the three variables given by queue length and the states of the arrival and service processes at the end of the time interval of length $\Delta t$, Haßlinger inspected the queue length $L$ and the time $n$ until the next arrival or next departure, only at time slots when an arrival occurs or a service is completed. Thus Haßlinger only needed to use a 2-dimensional state representation instead of the 3-dimensional state representation used in this paper. An important assumption used by Haßlinger is that the stationary probability of $(L, n)$ is given by $\alpha c^{L} P_{n}$ where $\alpha$ and $c$ are parameters, and $P_{n}$ is another parameter which depends on $n$.

A major contribution of this paper is the introduction of an easily implementable algorithm for solving the equations for the stationary probabilities of the queue length and the states of the arrival and service processes. This algorithm may be used to investigate other queuing systems which cannot be represented as continuous-time Markov chains. For example, Yang et al. [13] studied a model given by an unreliable $M / M / 1$ queue with a multistate server whose service rate deteriorates due to the shocks which occur randomly with random magnitudes. This algorithm has been successfully used to investigate the model given in Yang et al. [13] with the distribution of the interarrival time or service time changed to a fairly general distribution (see [14]).

\section{Derivation of Equations for the Stationary Probabilities}

In this section, a set of equations for the stationary probabilities of the queue length and the states of the arrival and service processes in the discretized CAR/CAR/1 queue is derived. First let $g(t)$ be the probability density function (pdf) of the service time and $\tau_{k}$ the interval $((k-1) \Delta t, k \Delta t]$ for $k=$ $0,1,2, \ldots$. Furthermore let

$$
\mu_{k}=\frac{g(k \Delta t)}{\int_{k \Delta t}^{\infty} g(u) d u}, \quad 1 \leq k \leq I,
$$

where $I$ is large enough such that

$$
\mu_{I} \cong \lim _{k \rightarrow \infty} \mu_{k}
$$

Suppose a service starts at time $t=0$. Then, for sufficiently small $\Delta t$, the probability that the service will be completed in the interval $\tau_{1}$ is approximately $\mu_{1} \Delta t$, and, given that the service is not completed in $\tau_{1}, \tau_{2}, \ldots, \tau_{k-1}$, the probability that the service will be completed in $\tau_{k}$ is approximately $\mu_{k} \Delta t, k=$ $2,3,4, \ldots$ where $\mu_{k}=\mu_{I}$ for $k \geq I$.

For the arrival process, let $f(t)$ be the pdf of the arrival time. Furthermore let

$$
\lambda_{k}=\frac{f(k \Delta t)}{\int_{k \Delta t}^{\infty} f(u) d u}, \quad 1 \leq k \leq J,
$$

where $J$ is large enough such that

$$
\lambda_{J} \cong \lim _{k \rightarrow \infty} \lambda_{k}
$$

Suppose a customer has arrived at time $t=0$. Then the next customer will arrive in the interval $\tau_{1}$ with an approximate probability $\lambda_{1} \Delta t$, and, given that the next customer does not arrive in the interval $\tau_{1}, \tau_{2}, \ldots, \tau_{k-1}$, the probability that the next customer will arrive in $\tau_{k}$ will be approximately $\lambda_{k} \Delta t$ for $k=2,3,4, \ldots$ where $\lambda_{k}=\lambda_{J}$ for $k \geq J$.

Given that a service starts at a time in $\tau_{0}$, we may define the state number $\xi_{k}$ of the service process at the end of $\tau_{k}$ as

$$
\xi_{k}= \begin{cases}0, & \text { if } \\ & \text { (i) } k=0 \text {; or } \\ & \text { (ii) the service ends in } \tau_{k}, \text { for } k \geq 1 ; \\ & \text { or } \\ & \text { (iii) the server is idle in } \tau_{k}, \\ \min (k, I), & \text { if the service does not end in } \tau_{k}, \\ & k \geq 1 .\end{cases}
$$

Next, given that a customer arrives at a time in $\tau_{0}$, we may define the state number $\psi_{k}$ of the arrival process at the end of $\tau_{k}$ as

$$
\psi_{k}= \begin{cases}0, & \text { if } k=0 \text { or the next customer } \\ & \text { arrives in } \tau_{k}, k \geq 1, \\ \min (k, J), & \text { if the next customer does not } \\ & \text { arrive in } \tau_{k}, k \geq 1 .\end{cases}
$$

Let $n_{k}$ be the queue length at the end of $\tau_{k}$ and $\mathbf{h}_{k}=$ $\left(n_{k}, \xi_{k}, \psi_{k}\right)$. We may refer to $\mathbf{h}_{k}$ as the vector of characteristics of the queue at the end of $\tau_{k}$.

Let $P_{n i j}^{(k)}$ be the probability that, at the end of $\tau_{k}$, the number of customers in the system is $n$ (including the customer that is being served), the service process is in state $i$, and the arrival process is in state $j$, where $n \geq 0, i \in\{0,1,2, \ldots, I\}$ and $j \in\{0,1,2, \ldots, J\}$. Assume that

$$
P_{n i j}=\lim _{k \rightarrow \infty} P_{n i j}^{(k)}
$$

exists. To find the $P_{n i j}$, we first make the following observations. 
Suppose at the end of $\tau_{k-1}$ the system is not empty, and the service and arrival processes are in state $i-1$ and $j-1$ at the end of $\tau_{k-1}$, respectively. Then only one of the following events can occur in the next time interval $\tau_{k}$ :

(a) a customer enters the system with the arrival rate $\lambda_{j^{*}}$, and, at the end of $\tau_{k}$, the vector of characteristics becomes $\mathbf{h}_{k}=\left(n+1, i^{*}, 0\right)$;

(b) a customer leaves the system with the departure rate $\mu_{i^{*}}$, and $\mathbf{h}_{k}=\left(n-1,0, j^{*}\right)$

(c) no customers enter or leave the system, and $\mathbf{h}_{k}=$ $\left(n, i^{*}, j^{*}\right)$.

Here, $i^{*}=\min (i, I)$ and $j^{*}=\min (j, J)$. However if the system is empty at the end of $\tau_{k-1}$, and the arrival process is in state $j-1$, then either one of the following events may occur in $\tau_{k}$

(d) a customer enters the system with arrival rate $\lambda_{j^{*}}$, and $\mathbf{h}_{k}=(1,0,0)$;

(e) no customers enter the system, and $\mathbf{h}_{k}=\left(0,0, j^{*}\right)$.

By setting $n_{k-1}=1, i-1=j-1=0$ and letting event (b) occurs in $\tau_{k}$, we get

$$
P_{001}^{(k)} \cong P_{100}^{(k-1)}\left(\mu_{1} \Delta t\right)\left(1-\lambda_{1} \Delta t\right) .
$$

When $k \rightarrow \infty$, (8) yields,

$$
P_{001} \cong P_{100}\left(\mu_{1} \Delta t\right)\left(1-\lambda_{1} \Delta t\right) .
$$

In general, for a given value of $\mathbf{h}_{k}$, we can likewise find the combinations of $\mathbf{h}_{k-1}$ and the event in $\tau_{k}$ which lead to $\mathbf{h}_{k}$, and obtain an equation similar to (9). Thus we can obtain the following equations:

$$
\begin{aligned}
P_{00 j} \cong & P_{00(j-1)}\left(1-\lambda_{j} \Delta t\right) \\
& +\sum_{i=0}^{j-1} P_{1 i(j-1)}\left(\mu_{i+1} \Delta t\right)\left(1-\lambda_{j} \Delta t\right) \\
& \text { for } 2 \leq j \leq J-1, \\
P_{00 J} \cong & P_{00(J-1)}\left(1-\lambda_{J} \Delta t\right)+P_{00 J}\left(1-\lambda_{J} \Delta t\right) \\
& +\sum_{i=0}^{J-1} P_{1 i(J-1)}\left(\mu_{i+1} \Delta t\right)\left(1-\lambda_{J} \Delta t\right) \\
& +\sum_{i=0}^{J-1} P_{1 i J}\left(\mu_{i+1} \Delta t\right)\left(1-\lambda_{J} \Delta t\right) \\
& +P_{1 I J}\left(\mu_{I} \Delta t\right)\left(1-\lambda_{J} \Delta t\right) .
\end{aligned}
$$

When the queue length is $n=1$,

$$
\begin{array}{r}
P_{n 00} \cong \sum_{j=1}^{J-1} P_{00 j}\left(\lambda_{j+1} \Delta t\right)+P_{00 J}\left(\lambda_{J} \Delta t\right), \\
P_{n 01} \cong \sum_{i=1}^{I-1} P_{(n+1) i 0}\left(\mu_{i+1} \Delta t\right)\left(1-\lambda_{1} \Delta t\right) \\
\quad+P_{(n+1) I 0}\left(\mu_{I} \Delta t\right)\left(1-\lambda_{1} \Delta t\right),
\end{array}
$$

$$
\begin{aligned}
& P_{n 0 j} \cong \sum_{i=0, i \neq j-1}^{I-1} P_{(n+1) i(j-1)}\left(\mu_{i+1} \Delta t\right)\left(1-\lambda_{j} \Delta t\right) \\
& +P_{(n+1) I(j-1)}\left(\mu_{I} \Delta t\right)\left(1-\lambda_{j} \Delta t\right) \\
& \text { for } 2 \leq j \leq J-1 \text {, } \\
& P_{n 0 J} \cong \sum_{i=0, i \neq J-1}^{I-1} P_{(n+1) i(J-1)}\left(\mu_{i+1} \Delta t\right)\left(1-\lambda_{J} \Delta t\right) \\
& +P_{(n+1) I(J-1)}\left(\mu_{I} \Delta t\right)\left(1-\lambda_{J} \Delta t\right) \\
& +\sum_{i=0}^{I-1} P_{(n+1) i J}\left(\mu_{i+1} \Delta t\right)\left(1-\lambda_{J} \Delta t\right) \\
& +P_{(n+1) I J}\left(\mu_{I} \Delta t\right)\left(1-\lambda_{J} \Delta t\right), \\
& P_{n i j} \cong P_{n(i-1)(j-1)}\left(1-\mu_{i} \Delta t\right)\left(1-\lambda_{j} \Delta t\right) \\
& \text { for } i \leq j, \quad 1 \leq i \leq I-1, \\
& 1 \leq j \leq J-1, \\
& P_{n i J} \cong P_{n(i-1)(J-1)}\left(1-\mu_{i} \Delta t\right)\left(1-\lambda_{J} \Delta t\right) \\
& +P_{n(i-1) J}\left(1-\mu_{i} \Delta t\right)\left(1-\lambda_{J} \Delta t\right) \\
& \text { for } 1 \leq i \leq I-1 \text {, } \\
& P_{n I J} \cong P_{n(I-1)(J-1)}\left(1-\mu_{I} \Delta t\right)\left(1-\lambda_{J} \Delta t\right) \\
& +P_{n(I-1) J}\left(1-\mu_{I} \Delta t\right)\left(1-\lambda_{J} \Delta t\right) \\
& +P_{n I J}\left(1-\mu_{I} \Delta t\right)\left(1-\lambda_{J} \Delta t\right) \text {. }
\end{aligned}
$$

When the queue length is $n=2$, the expressions for $P_{n 0}, 1 \leq$ $j \leq J$, are the same as those given by (13), (14), and (15). Other $P_{n i j}$ when the queue length is $n=2$ can be computed from the equations below:

$$
\begin{array}{r}
P_{n i 0} \cong \sum_{j \geq i-1}^{J-1} P_{(n-1)(i-1) j}\left(1-\mu_{i} \Delta t\right)\left(\lambda_{j+1} \Delta t\right) \\
+P_{(n-1)(i-1) J}\left(1-\mu_{i} \Delta t\right)\left(\lambda_{J} \Delta t\right) \\
\quad \text { for } 1 \leq i \leq I-1, \\
P_{n I 0} \cong P_{(n-1)(I-1)(J-1)}\left(1-\mu_{I} \Delta t\right)\left(\lambda_{J} \Delta t\right) \\
+P_{(n-1)(I-1) J}\left(1-\mu_{I} \Delta t\right)\left(\lambda_{J} \Delta t\right) \\
+P_{(n-1) I J}\left(1-\mu_{I} \Delta t\right)\left(\lambda_{J} \Delta t\right), \\
P_{n i j} \cong P_{n(i-1)(j-1)}\left(1-\mu_{i} \Delta t\right)\left(1-\lambda_{j} \Delta t\right) \\
\text { for } i \neq j, \quad 1 \leq i \leq I-1, \\
\quad 1 \leq j \leq J-1,
\end{array}
$$




$$
\begin{aligned}
P_{n i J} \cong & P_{n(i-1)(J-1)}\left(1-\mu_{i} \Delta t\right)\left(1-\lambda_{J} \Delta t\right) \\
& +P_{n(i-1) J}\left(1-\mu_{i} \Delta t\right)\left(1-\lambda_{J} \Delta t\right) \\
& \text { for } 1 \leq i \leq I-1, \\
P_{n I j} \cong & P_{n(I-1)(j-1)}\left(1-\mu_{I} \Delta t\right)\left(1-\lambda_{j} \Delta t\right) \\
& +P_{n I(j-1)}\left(1-\mu_{I} \Delta t\right)\left(1-\lambda_{j} \Delta t\right) \\
& \text { for } 1 \leq j \leq J-1, \\
P_{n I J} \cong & P_{n(I-1) J}\left(1-\mu_{I} \Delta t\right)\left(1-\lambda_{J} \Delta t\right) \\
& +P_{n I(J-1)}\left(1-\mu_{I} \Delta t\right)\left(1-\lambda_{J} \Delta t\right) \\
& +P_{n I J}\left(1-\mu_{I} \Delta t\right)\left(1-\lambda_{J} \Delta t\right) .
\end{aligned}
$$

When the queue length is $n \geq 3$, the values of all the $P_{n i j}$ (except $P_{n i 0}$ ) can be computed using (21) to (24) and (13) to (15), whereas those of $P_{n i 0}$ can be computed using the following equations:

$$
\begin{gathered}
P_{n 10} \cong \sum_{j=1}^{J-1} P_{(n-1) 0 j}\left(1-\mu_{1} \Delta t\right)\left(\lambda_{j+1} \Delta t\right) \\
+P_{(n-1) 0 J}\left(1-\mu_{1} \Delta t\right)\left(\lambda_{J} \Delta t\right), \\
P_{n i 0} \cong \sum_{j=0, j \neq i-1}^{J-1} P_{(n-1)(i-1) j}\left(1-\mu_{i} \Delta t\right)\left(\lambda_{j+1} \Delta t\right) \\
\quad+P_{(n-1)(i-1) J}\left(1-\mu_{i} \Delta t\right)\left(\lambda_{J} \Delta t\right) \\
P_{n I 0} \cong \sum_{j=0, j \neq I-1}^{J-1} P_{(n-1)(I-1) j}\left(1-\mu_{I} \Delta t\right)\left(\lambda_{j+1} \Delta t\right) \\
+P_{(n-1)(I-1) J}\left(1-\mu_{I} \Delta t\right)\left(\lambda_{J} \Delta t\right) \\
+\sum_{j=0}^{J-1} P_{(n-1) I j}\left(1-\mu_{I} \Delta t\right)\left(\lambda_{j+1} \Delta t\right) \\
+P_{(n-1) I J}\left(1-\mu_{I} \Delta t\right)\left(\lambda_{J} \Delta t\right) .
\end{gathered}
$$

\section{Stationary Queue Length Distribution}

Before solving (9) to (27) in Section 2, to obtain the stationary queue length distribution, we may first let $c_{i j}, d_{i j}, e_{i j}, f_{j}$ and $g_{i j}$ be constants and introduce the following notations:

(a) $P_{n^{* *}}=\left\{P_{n i j}: 0 \leq i \leq I, 0 \leq j \leq J\right\}$;

(b) $\left(P_{m^{* *}}, P_{(m+1)^{* *}}, P_{(m+2)^{* *}}\right)$ denotes the set of equations of the form

$$
\begin{gathered}
\sum_{i=0}^{I} \sum_{j=0}^{J} c_{i j} P_{m i j}+\sum_{i=0}^{I} \sum_{j=0}^{J} d_{i j} P_{(m+1) i j} \\
+\sum_{i=0}^{I} \sum_{j=0}^{J} e_{i j} P_{(m+2) i j} \cong 0
\end{gathered}
$$

(c) $\left(P_{m i j} \mid P_{0^{* *}}, P_{(m+1)^{* *}}\right)$ denotes the equation of the form

$$
P_{m i j} \cong \sum_{j=0}^{J} f_{j} P_{00 j}+\sum_{i=0}^{I} \sum_{j=0}^{J} g_{i j} P_{(m+1) i j} .
$$

With the above notations, (12) to (18) in the case when $n=1$ can be represented as

$$
\left(P_{0^{* *}}, P_{1^{* *}}, P_{2^{* *}}\right)
$$

and (19) to (24) together with (13) to (15) in the case when $n=2$ may be represented as

$$
\left(P_{1^{* *}}, P_{2^{* *}}, P_{3^{* *}}\right)
$$

Furthermore (25) to (27) together with (21) to (24) and (13) to (15) in the case when $n \geq 3$ may be represented as

$$
\left(P_{(n-1)^{* *}}, P_{n^{* *}}, P_{(n+1)^{* *}}\right) .
$$

It can be shown that, from the set of equations given by (30), we can get

$$
\left(P_{1 i j} \mid P_{0^{* *}}, P_{2^{* *}}\right) \quad \text { for } 0 \leq i \leq I, 0 \leq j \leq J .
$$

By substituting the expression of $P_{1 i j}$ given by (33) into (31), and solving for $P_{2 i j}$, we get

$$
\left(P_{2 i j} \mid P_{0^{* *}}, P_{3^{* *}}\right) \quad \text { for } 0 \leq i \leq I, 0 \leq j \leq J .
$$

By substituting the expression of $P_{2 i j}$ given by (34) into (32) when $n=3$ and solving for $P_{3 i j}$, we get

$$
\left(P_{3 i j} \mid P_{0^{* *}}, P_{4^{* *}}\right) \quad \text { for } 0 \leq i \leq I, 0 \leq j \leq J .
$$

Next for $n \geq 4$, repeat the process of substituting the expression of $P_{(n-1) i j}$ given by

$$
\left(P_{(n-1) i j} \mid P_{0^{* *}}, P_{n^{* *}}\right),
$$

into (32) and solving for $P_{n i j}$ to get

$$
\left(P_{n i j} \mid P_{0^{* *}}, P_{(n+1)^{* *}}\right) \quad \text { for } 0 \leq i \leq I, 0 \leq j \leq J .
$$

When $n=N$ is large enough, we may set all the $P_{(n+1) i j}$ in (37) to be zero and obtain

$$
\left(P_{N i j} \mid P_{0^{* *}}\right) \text { for } 0 \leq i \leq I, 0 \leq j \leq J .
$$

Substituting (38) into (37) when $n=N-1$, we get

$$
\left(P_{(N-1) i j} \mid P_{0^{* *}}\right) \quad \text { for } 0 \leq i \leq I, 0 \leq j \leq J .
$$

Similarly, for $n=N-2, N-3, \ldots, 1$, we may perform the substitution of $\left(P_{(n+1) i j} \mid P_{0^{* *}}\right)$ into (37) and obtain

$$
\left(P_{n i j} \mid P_{0^{* *}}\right) \text { for } 0 \leq i \leq I, 0 \leq j \leq J \text {. }
$$


When $n=1,(40)$ yields $\left(P_{1 i j} \mid P_{0^{* *}}\right)$. By using the results given by $\left(P_{1 i j} \mid P_{0^{* *}}\right)$ and (9) to (11), we get the following system of $J$ equations:

$$
\left(P_{00 j} \mid P_{0^{* *}}\right) \quad \text { for } 0 \leq j \leq J
$$

An inspection of (41) reveals that, among the $J$ equations, only $J-1$ of them are linearly independent. Hence, we need to include another linearly independent equation so that the resulting system of $J$ equations has a unique solution. Equating the sum of the left sides of the equations given by (40) to the sum of the right sides of (40), we get an equation of the form,

$$
\sum_{n=1}^{N} \sum_{i} \sum_{j} P_{n i j}=\sum_{j} k_{j} P_{00 j}
$$

where the $k_{j}$ are constants.

As $\sum_{n=0}^{N} \sum_{i} \sum_{j} P_{n i j} \cong 1$, we get from (42) an equation involving only $P_{00}, 1 \leq j \leq J$. The equation derived from (42) and $J-1$ equations chosen from (41) constitute a system of $J$ equations which can be solved to yield numerical answers for $P_{00 j}, 1 \leq j \leq J$. Then using (40), we can get numerical answers for $P_{n i j}$ where $n \geq 1,0 \leq i \leq I$, and $0 \leq j \leq J$. The stationary probability that the queue length is $n$ can then be obtained as

$$
P_{n}=\sum_{i=0}^{I} \sum_{j=0}^{J} P_{n i j}
$$

\section{Waiting Time Distribution}

Suppose a customer arrives at the system which is in the stationary state. Let the time of arrival of the customer be denoted as $t=0$. Furthermore, let $W_{q}$ be the time the customer needs to wait before being served and

$$
W_{q}(t)=P\left(W_{q} \leq t\right)
$$

the cumulative distribution function (cdf) of the waiting time $W_{q}$.

To find the waiting time distribution $W_{q}(t)$, we first note that when the system is in the stationary state, an arrival of a customer at time $t=0$ which is inside an interval $\tau$ of length $\Delta t$ may occur with an approximate probability $\lambda_{j+1} \Delta t$ if the arrival process is in state $j$ at the beginning of the interval $\tau$. Meanwhile at the beginning of $\tau$, the service process may be in state $i$ where $0 \leq i \leq I$. Thus the probability that

(i) the queue length at the beginning of $\tau$ is $n$,

(ii) the service process is in state $i$ at the beginning of $\tau$,

(iii) the arrival process is in state $j$ at the beginning of $\tau$,

(iv) a customer arrives in $\tau$

is given approximately by

$$
P_{n i j} \lambda_{j+1} \Delta t
$$

Let $g_{i+1}(t)$ be the pdf of the service time given that the service process is in state $i$ at the beginning of $\tau$, and $g^{(n-1)}(t)$ the $(n-1)$-fold convolution of $g(t)$. The customer who arrives in $\tau$ (see (iv)) under the conditions given by (i), (ii), and (iii) above will have a waiting time of zero if $n=0$, and a waiting time in which the pdf is given by the convolution $g_{i+1}(t) *$ $g^{(n-1)}(t)$ if $n \geq 1$. The cdf $W_{q}(t)$ is then given approximately by

$$
\begin{aligned}
& W_{q}(t) \\
& \cong\left(\sum_{j=1}^{J} P_{00 j} \lambda_{j+1} \Delta t\right. \\
& \left.\quad+\sum_{n=1}^{N} \sum_{i=0}^{I} \sum_{j=0}^{J} P_{n i j} \lambda_{j+1} \Delta t \int_{u=0}^{t} g_{i+1}(u) * g^{(n-1)}(u) d u\right) \\
& \quad \times\left(\sum_{j=1}^{J} P_{00 j} \lambda_{j+1} \Delta t+\sum_{n=1}^{N} \sum_{i=0}^{I} \sum_{j=0}^{J} P_{n i j} \lambda_{j+1} \Delta t\right)^{-1} .
\end{aligned}
$$

The cdf $W_{q}(t)$ may also be computed approximately by a simulation procedure described below.

Suppose a customer arrives at time $t=0$ and the next $m$ th customer arrives at time $t=\sum_{k=1}^{m} A_{k}$, where $A_{1}, A_{2}, \ldots$ are independent and identically distributed with pdf $f(t)$. Next let the service time of the next $m$ th customer be $B_{m}$ of which $B_{0}, B_{1}, \ldots$ are independent and identically distributed with pdf $g(t)$. For a chosen large integer $M$, the value of $\underline{v}=\left\{\left(0, B_{0}\right),\left(A_{1}, B_{1}\right), \ldots,\left(A_{M}, B_{M}\right)\right\}$ is generated and the following waiting times are obtained:

$$
\begin{aligned}
& W_{q, 0}=0, \\
& W_{q, m}= \begin{cases}0, & \text { if } W_{q, m-1}+B_{m-1}<A_{m}, \\
W_{q, m-1}+B_{m-1}-A_{m}, & \text { if } W_{q, m-1}+B_{m-1} \geq A_{m}, \\
& 1 \leq m \leq M-1,\end{cases}
\end{aligned}
$$

where $W_{q, m}$ is the waiting time of the $m$ th customer. Then

$$
W_{q}(t) \cong \frac{\text { Number of the } W_{q, m} \text { which are less than } t}{M} \text {. }
$$

\section{Numerical Examples}

Let $\operatorname{Gamma}(\kappa, \theta)$ denote a gamma distribution of which $\kappa$ is the shape parameter and $\theta$ is the scale parameter. The related probability density function is then given by $f(t ; \kappa, \theta)=\left(t^{\kappa-1} e^{-t / \theta}\right) /\left(\theta^{\kappa} \Gamma(\kappa)\right)$. Consider an example in which the service time $(S)$ has a gamma distribution with the parameter vector $\left(\kappa_{1}, \theta_{1}\right)=(1.5,2)$, and the interarrival time $(T)$ has another gamma distribution with the parameter vector $\left(\kappa_{2}, \theta_{2}\right)=(3.1,2)$. The utilization factor will then be $\rho=E(S) / E(T)=0.48$. The reason for considering 
gamma distribution $(\kappa, \theta)$ with fractional values of the shape parameter $\kappa$ is that the term $t^{\kappa-1}$ appearing in the pdf $f(t)$ and $g(t)$ will usually make the existing analytical methods for finding queue length distribution fail. The reason behind such failure is that when we set $t=x+y$, the function $(x+y)^{\kappa-1}$ cannot be expressed as a finite sum of products of a function of $x$ alone and a function of $y$ alone.

The following is a procedure to find the values of $\Delta t$ and $I$ (or $J$ ). Initially we find the value of $T$ such that the rates at time $t \geq T$ exhibit small variations. A small fractional value (e.g., 0.1 or 0.05 ) is assigned to $\Delta t$ and $I$ is then obtained as the integer which is approximately equal to $T / \Delta t$. If $I$ is very large (e.g., $I>1000$ ), then a bigger unit is chosen for $t$ until $I \leq 1000$. It can be shown that when $\Delta t=0.04$, suitable values of $I$ and $J$ are, respectively, $I=550$ and $J=550$. By using the proposed numerical method, the stationary queue length distribution is found. The stationary queue length distribution may also be computed using the simulation procedure in the software "QtsPlus" (accompanying software for Gross and Harris [15]) when the number of runs is $M_{1}=107$. The results obtained are shown in Table 1.

From Table 1, we see that the stationary queue length distribution obtained using the proposed numerical method is close to that obtained from the software "QtsPlus."

Table 2 shows the stationary queue length probabilities found by the numerical method using $N=30$. The reason for choosing $N=30$ is that, for each given value of $\Delta t$, the value of $P_{30}$ will then be of the order of about $10^{-20}$ which is small enough for us to get, for $0 \leq n \leq 7$, a value of $P_{n}$ which is accurate up to the 6 decimal points. For a given value $n$ of the queue length, we may use the values in the columns under $\Delta t$ and $n$ in Table 2 to fit a polynomial of low degree in $\Delta t$ to the queue length probability. The value given by the fitted polynomial when $\Delta t=0$ will represent the final result based on the numerical method for the queue length probability. It can be shown that, for all the values of $n$ considered, the fitted polynomials are all very satisfactory. Thus the final results at $\Delta t=0$ would be quite accurate. Table 2 shows that the numerical results for queue length $n=0,2,3$, and 4 agree quite well with those based on "QtsPlus." Meanwhile the numerical results for queue length $n=1,5,6$, and 7 are somewhat more accurate than those based on simulation.

Table 3 shows that the stationary waiting time distribution obtained by using the numerical method in Section 4 is close to that obtained by the simulation procedure.

\section{Discrete Time GI/G/1 Queue}

The stationary queue length and stationary waiting time distributions of a discrete time GI/G/1 can also be found by using the proposed numerical method in Sections 2 to 4 after some modifications of the equations for the stationary probabilities given in Section 2. An explanation of why the above modifications are necessary is as follows.

First we note that the values of the $\mu_{k}$ (or $\lambda_{k}$ ) for the discrete service time (or arrival time) distribution are such
TABLE 1: Comparison of stationary queue length distribution computed from the proposed numerical method, and that obtained from the software "QtsPlus" $\left[\left(\kappa_{1}, \theta_{1}\right)=(1.5,2),\left(\kappa_{2}, \theta_{2}\right)=(3.1,2), \Delta t=0.04\right]$.

\begin{tabular}{lcc}
\hline \multirow{2}{*}{ Queue length, $n$} & \multicolumn{2}{c}{$P$ (queue length $=n)$} \\
& Numerical method & Simulation (QtsPlus) \\
\hline 0 & 0.518854 & 0.516033 \\
1 & 0.366244 & 0.366475 \\
2 & 0.089790 & 0.091326 \\
3 & 0.019748 & 0.020488 \\
4 & 0.004227 & 0.004444 \\
5 & $8.97 E-04$ & $9.48 E-04$ \\
6 & $1.90 E-04$ & $2.15 E-04$ \\
7 & $4.01 E-05$ & $5.44 E-05$ \\
8 & $8.47 E-06$ & $1.33 E-05$ \\
9 & $1.79 E-06$ & $3.43 E-06$ \\
10 & $3.78 E-07$ & $1.79 E-07$ \\
$\vdots$ & $\vdots$ & $\vdots$ \\
20 & $6.67 E-14$ & 0 \\
\hline
\end{tabular}

that all the $\mu_{k}$ (or $\lambda_{k}$ ) are zero except for the cases when $k \Delta t$ coincides with the service time (or arrival time) which has a nonzero probability of occurrence. Let the values of such $k$ be denoted by $k_{1}, k_{2}, \ldots, k_{d}$. The value of a typical $\lambda_{k_{i}}$ will be such that $\lambda_{k_{i}} \Delta t$ is a constant. This means that when $\Delta t$ is made very small, the value of $\lambda_{k_{i}}$ will have to be inflated correspondingly. Thus, if the system is not empty at time $t$, the simultaneous occurrence of the events that

(A) a customer arrives within the interval $(t, t+\Delta t]$, and

(B) a service is completed within the interval $(t, t+\Delta t]$

may not tend to zero when $\Delta t$ tends to zero. Thus the equations for stationary probabilities given in Section 2 need to be modified by taking into account of the simultaneous occurrence of events (A) and (B). The modified version of the equations in Section 2 is as follows.

When the queue length is $n=0$, the values of the $P_{n i j}$ can be found from (9)-(11). When the queue length is $n=1$, the expressions for $P_{n i j}, 1 \leq j \leq J, i \leq j$ are the same as those given by (16)-(18), whereas $P_{n 0 j}$ can be computed from the equations as follows:

$$
\begin{aligned}
P_{n 00} \cong & \sum_{j=1}^{J-1} P_{00 j}\left(\lambda_{j+1} \Delta t\right)+P_{00 J}\left(\lambda_{J} \Delta t\right) \\
& +\sum_{i=0}^{I-1} \sum_{j \geq i}^{J-1} P_{n i j}\left(\mu_{i+1} \Delta t\right)\left(\lambda_{j+1} \Delta t\right) \\
& +\sum_{i=0}^{I-1} P_{n i J}\left(\mu_{i+1} \Delta t\right)\left(\lambda_{J} \Delta t\right)+P_{n I J}\left(\mu_{I} \Delta t\right)\left(\lambda_{J} \Delta t\right),
\end{aligned}
$$


TABLE 2: Comparison of stationary queue length probabilities found by the numerical method using various other values of $\Delta t$, the extrapolated values, and those obtained from the software "QtsPlus" $\left(\kappa_{1}, \theta_{1}\right)=(1.5,2),\left(\kappa_{2}, \theta_{2}\right)=(3.1,2)$.

\begin{tabular}{|c|c|c|c|c|c|c|c|c|}
\hline \multirow{2}{*}{$\Delta t$} & \multicolumn{8}{|c|}{ Queue length } \\
\hline & 0 & 1 & 2 & 3 & 4 & 5 & 6 & 7 \\
\hline 0.088000 & 0.521712 & 0.366372 & 0.088018 & 0.018916 & 0.003952 & 0.000818 & 0.000169 & $3.47808 E-05$ \\
\hline 0.073333 & 0.520874 & 0.366316 & 0.088550 & 0.019165 & 0.004034 & 0.000841 & 0.000175 & $3.63184 E-05$ \\
\hline 0.062857 & 0.520258 & 0.366284 & 0.088935 & 0.019345 & 0.004093 & 0.000858 & 0.000179 & $3.74598 E-05$ \\
\hline 0.059459 & 0.520054 & 0.366276 & 0.089060 & 0.019405 & 0.004113 & 0.000864 & 0.000181 & $3.78380 E-05$ \\
\hline 0.055000 & 0.519784 & 0.366266 & 0.089226 & 0.019482 & 0.004139 & 0.000871 & 0.000183 & $3.83000 E-05$ \\
\hline 0.051163 & 0.519550 & 0.366258 & 0.089369 & 0.019550 & 0.004161 & 0.000878 & 0.000185 & $3.87789 E-05$ \\
\hline 0.048889 & 0.519410 & 0.366255 & 0.089454 & 0.019590 & 0.004174 & 0.000882 & 0.000186 & $3.90411 E-05$ \\
\hline 0.040000 & 0.518854 & 0.366244 & 0.089790 & 0.019748 & 0.004227 & 0.000897 & 0.000190 & $4.00846 E-05$ \\
\hline 0.037931 & 0.518722 & 0.366243 & 0.089868 & 0.019785 & 0.004239 & 0.000900 & 0.000191 & $4.03318 E-05$ \\
\hline 0.033846 & 0.518461 & 0.366241 & 0.090024 & 0.019859 & 0.004264 & 0.000908 & 0.000193 & $4.08250 E-05$ \\
\hline 0.031429 & 0.518304 & 0.366241 & 0.090117 & 0.019902 & 0.004279 & 0.000912 & 0.000194 & $4.11199 E-05$ \\
\hline 0.027848 & 0.518070 & 0.366241 & 0.090254 & 0.019968 & 0.004300 & 0.000918 & 0.000195 & $4.15611 E-05$ \\
\hline 0.027500 & 0.518047 & 0.366242 & 0.090268 & 0.019974 & 0.004302 & 0.000919 & 0.000196 & $4.16043 E-05$ \\
\hline Fitted value & 0.516162 & 0.366307 & 0.091340 & 0.020486 & 0.004474 & 0.000969 & 0.000210 & $4.51599 E-05$ \\
\hline Value based on simulation & 0.516033 & 0.366475 & 0.091326 & 0.020488 & 0.004444 & 0.000948 & 0.000215 & $5.43826 E-05$ \\
\hline
\end{tabular}

$$
\begin{array}{r}
P_{n 0 j} \cong \sum_{i=0}^{I-1} P_{(n+1) i(j-1)}\left(\mu_{i+1} \Delta t\right)\left(1-\lambda_{j} \Delta t\right) \\
+P_{(n+1) I(j-1)}\left(\mu_{I} \Delta t\right)\left(1-\lambda_{j} \Delta t\right) \\
\quad \text { for } 1 \leq j \leq J-1, \\
P_{n 0 J} \cong \sum_{i=0}^{I-1} P_{(n+1) i(J-1)}\left(\mu_{i+1} \Delta t\right)\left(1-\lambda_{J} \Delta t\right) \\
+P_{(n+1) I(J-1)}\left(\mu_{I} \Delta t\right)\left(1-\lambda_{J} \Delta t\right) \\
+\sum_{i=0}^{I-1} P_{(n+1) i J}\left(\mu_{i+1} \Delta t\right)\left(1-\lambda_{J} \Delta t\right) \\
\quad+P_{(n+1) I J}\left(\mu_{I} \Delta t\right)\left(1-\lambda_{J} \Delta t\right) .
\end{array}
$$

When $n=2$, the values of the $P_{n 0 j}, 1 \leq j \leq J$ can be computed using (50)-(51), while (19)-(20) can be used to find the values of $P_{n i 0}, 1 \leq i \leq I$. The values of the $P_{n i J}$ and $P_{n I j}$ can be obtained from (22) and (23), respectively. All the other values of $P_{n i j}$ can be computed using the following equations:

$$
\begin{aligned}
P_{n 00} \cong & \sum_{i=0}^{I-1} \sum_{j=0}^{J-1} P_{n i j}\left(\mu_{i+1} \Delta t\right)\left(\lambda_{j+1} \Delta t\right) \\
& +\sum_{i=0}^{I-1} P_{n i J}\left(\mu_{i+1} \Delta t\right)\left(\lambda_{J} \Delta t\right) \\
& +\sum_{j=0}^{J-1} P_{n I j}\left(\mu_{I} \Delta t\right)\left(\lambda_{j+1} \Delta t\right)+P_{n I J}\left(\mu_{I} \Delta t\right)\left(\lambda_{J} \Delta t\right),
\end{aligned}
$$

TABLE 3: Comparison of stationary waiting time distribution computed, respectively, by using the proposed numerical method and the simulation procedure $(\Delta t=0.04)$.

\begin{tabular}{lcc}
\hline Time, $t$ & \multicolumn{2}{c}{$P\left(W_{q} \leq t\right)$} \\
\hline 0 & Numerical method & Simulation \\
0.04 & 0.716771 & 0.717751 \\
0.08 & 0.720011 & 0.719061 \\
0.12 & 0.723321 & 0.721774 \\
0.16 & 0.726602 & 0.725983 \\
0.20 & 0.729799 & 0.728072 \\
0.24 & 0.732959 & 0.731658 \\
0.28 & 0.735939 & 0.734215 \\
0.32 & 0.738872 & 0.738393 \\
0.36 & 0.741849 & 0.740451 \\
$\vdots$ & 0.744914 & 0.742415 \\
20 & $\vdots$ & $\vdots$ \\
\hline
\end{tabular}

$$
\begin{aligned}
P_{n i j} \cong & P_{n(i-1)(j-1)}\left(1-\mu_{i} \Delta t\right)\left(1-\lambda_{j} \Delta t\right) \\
& \text { for } 1 \leq i \leq I-1, \quad 1 \leq j \leq J-1, \\
P_{n I J} \cong & P_{n(I-1)(J-1)}\left(1-\mu_{I} \Delta t\right)\left(1-\lambda_{J} \Delta t\right) \\
& +P_{n(I-1) J}\left(1-\mu_{I} \Delta t\right)\left(1-\lambda_{J} \Delta t\right) \\
& +P_{n I(J-1)}\left(1-\mu_{I} \Delta t\right)\left(1-\lambda_{J} \Delta t\right) \\
& +P_{n I J}\left(1-\mu_{I} \Delta t\right)\left(1-\lambda_{J} \Delta t\right) .
\end{aligned}
$$


TABLE 4: Comparison of stationary queue length distribution computed using the proposed numerical method and that given in Kim and Chaudhry [11].

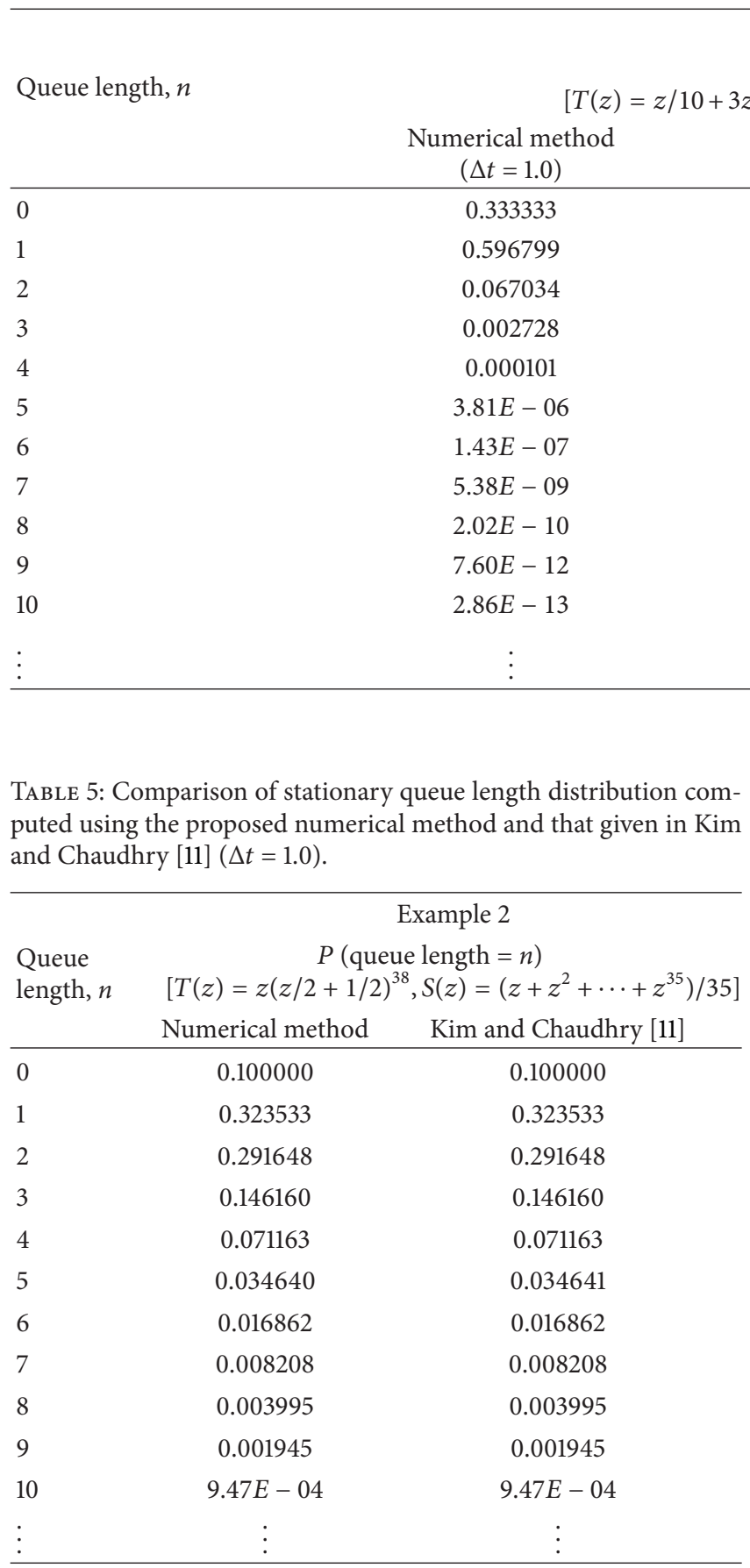

For $n \geq 3$, the values of all the $P_{n i j}$ (except $P_{n i 0}$ ) can be computed using (22)-(23) and (50)-(54), whereas those of $P_{n i 0}$ can be computed using the following equations:

$$
\begin{array}{r}
P_{n i 0} \cong \sum_{j=0}^{J-1} P_{(n-1)(i-1) j}\left(1-\mu_{i} \Delta t\right)\left(\lambda_{j+1} \Delta t\right) \\
+P_{(n-1)(i-1) J}\left(1-\mu_{i} \Delta t\right)\left(\lambda_{J} \Delta t\right) \\
\text { for } 1 \leq i \leq I-1,
\end{array}
$$

$$
\begin{array}{r}
P_{n I 0} \cong \sum_{j=0}^{J-1} P_{(n-1)(I-1) j}\left(1-\mu_{I} \Delta t\right)\left(\lambda_{j+1} \Delta t\right) \\
+P_{(n-1)(I-1) J}\left(1-\mu_{I} \Delta t\right)\left(\lambda_{J} \Delta t\right) \\
+\sum_{j=0}^{J-1} P_{(n-1) I j}\left(1-\mu_{I} \Delta t\right)\left(\lambda_{j+1} \Delta t\right) \\
+P_{(n-1) I J}\left(1-\mu_{I} \Delta t\right)\left(\lambda_{J} \Delta t\right) .
\end{array}
$$

We may solve the above equations by using the proposed numerical method in Section 3 to obtain all the values of $P_{n i j}$ and subsequently the stationary queue length distribution. From the values of the stationary probabilities, we can find the stationary waiting time distribution by using the method proposed in Section 4 . The cdf $W_{q}(t)$ for the discrete time $\mathrm{GI} / \mathrm{G} / 1$ queue is now given approximately by

$$
\begin{aligned}
& W_{q}(t) \\
& \cong\left(\sum_{j=1}^{J} P_{00 j} \lambda_{j+1} \Delta t\right. \\
& \quad+\sum_{n=1}^{N} \sum_{i=0}^{I} \sum_{j=0}^{J} P_{n i j}\left(\lambda_{j+1} \Delta t\right)\left(1-\mu_{i+1} \Delta t\right) \\
& \quad \times \int_{u=0}^{t} g_{i+1}(u) * g^{(n-1)}(u) d u \\
& \left.\quad+\sum_{n=1}^{N} \sum_{i=0}^{I} \sum_{j=0}^{J} P_{n i j}\left(\lambda_{j+1} \Delta t\right)\left(\mu_{i+1} \Delta t\right) \int_{u=0}^{t} g^{(n-1)}(u) d u\right) \\
& \quad \times\left(\sum_{j=1}^{J} P_{00 j} \lambda_{j+1} \Delta t+\sum_{n=1}^{N} \sum_{i=0}^{I} \sum_{j=0}^{J} P_{n i j} \lambda_{j+1} \Delta t\right)^{-1} .
\end{aligned}
$$


TABLE 6: Comparison of stationary queue length distributions computed using the proposed numerical method and that given in Kim and Chaudhry [11] $(\Delta t=1.0)$.

\begin{tabular}{|c|c|c|c|c|}
\hline \multirow{3}{*}{ Queue length, $n$} & \multicolumn{2}{|c|}{ Example 3} & \multicolumn{2}{|c|}{ Example 4} \\
\hline & \multicolumn{2}{|c|}{$\begin{aligned} & P(\text { Queue length }=n) \\
{[T(z)=} & \left.z(z / 2+1 / 2)^{38}, S(z)=\left(z+z^{2}+z^{3}\right) / 3\right]\end{aligned}$} & \multicolumn{2}{|c|}{$\begin{aligned} P(\text { Queue length } & =n) \\
{\left[T(z)=z(z / 2+1 / 2)^{38}, S(z)\right.} & =\left(z+z^{2}+\cdots+z^{19}\right) / 19\end{aligned}$} \\
\hline & Numerical method & Kim and Chaudhry [11] & Numerical method & Kim and Chaudhry [11] \\
\hline 0 & 0.900000 & 0.900000 & 0.500000 & 0.500000 \\
\hline 1 & 0.100000 & 0.100000 & 0.494811 & 0.494811 \\
\hline 2 & $2.49 E-12$ & $2.49 E-12$ & 0.005189 & 0.005189 \\
\hline 3 & $2.21 E-25$ & $2.21 E-25$ & $1.53 E-08$ & $1.53 E-08$ \\
\hline 4 & $1.52 E-46$ & 0 & $2.19 E-14$ & $2.19 E-14$ \\
\hline 5 & $3.54 E-60$ & 0 & $3.13 E-20$ & $2.75 E-20$ \\
\hline 6 & $\vdots$ & $\vdots$ & $\vdots$ & $\vdots$ \\
\hline
\end{tabular}

TABLE 7: Stationary waiting time distributions computed by using the proposed numerical method $(\Delta t=1.0)$.

\begin{tabular}{lcccc}
\hline \multirow{2}{*}{ Time, $t$} & \multicolumn{5}{c}{$P\left(W_{q} \leq t\right)$} \\
& Example 1 & Example 2 & Example 3 & Example 4 \\
\hline 0 & 0.847762 & 0.242019 & 1 & 0.955516 \\
1 & 0.964654 & 0.259177 & 1 & 0.972644 \\
2 & 0.992963 & 0.276887 & 1 & 0.984262 \\
3 & 0.998538 & 0.294817 & 1 & 0.991529 \\
4 & 0.999699 & 0.313157 & 1 & 0.995754 \\
5 & 0.999938 & 0.331907 & 1 & 0.998002 \\
6 & 0.999987 & 0.350904 & 1 & 0.999113 \\
7 & 0.999997 & 0.370697 & 1 & 0.999619 \\
8 & 0.999999 & 0.390268 & 1 & 0.999842 \\
9 & 1 & 0.410278 & 1 & 0.999933 \\
$\vdots$ & $\vdots$ & $\vdots$ & $\vdots$ & $\vdots$ \\
\hline
\end{tabular}

Table 4 shows the results of the stationary queue length distribution computed using the proposed numerical method. The table also shows the results given in [11] where the authors found the stationary queue length distribution from the sojourn time distribution using the distributional Little's law. The functions $T(z)$ and $S(z)$ appearing in Tables 4-6 and 8-9 are, respectively, the probability generating functions of the discrete service time and interarrival time.

From Table 4, we can see that the queue length probabilities obtained by using the proposed numerical method are close to those given in [11]. When $\Delta t \leq 1$, the values of the $\mu_{k}$ (or $\lambda_{k}$ ) are able to capture all the details of the discrete distribution of the service time (or arrival time). Thus the results given in columns 2 and 3 in Table 4 are identical.

Tables 5 and 6 show the stationary queue length distribution in three other examples of discrete queue.
Tables 5 and 6 show that the results obtained by using the proposed numerical method are very close to those given in [11].

From the stationary probabilities, the stationary waiting time distributions can be obtained using (57). The results obtained are shown in Table 7.

For a customer who arrives at time $t=0$, his sojourn time is equal to the sum of his waiting time and service time. Thus, from the waiting time and service time distributions of the incoming customer, we can compute his sojourn time distribution. Tables 8 and 9 show the results of the stationary sojourn time distribution computed using the proposed numerical method and those given in [11].

From Tables 8 and 9, we can see that the stationary sojourn time distributions computed by using the proposed numerical method are very close to those given in [11].

\section{Conclusion}

Most of the existing methods in the literature find the queue length distribution in the GI/G/1 queue via the waiting time distribution. On the contrary, the present proposed method finds the queue length distribution directly for the CAR/ $\mathrm{CAR} / 1$ queue. The accuracy of the numerical results for the distribution can be greatly improved by an extrapolation process. Furthermore the queue length distribution thus found can later be used to find the waiting time distribution and sojourn time distribution. The main drawback of the proposed method is that we may encounter dimensionality problem when $I$ (or $J$ ) is very large. For example, when the distribution of the interarrival time tends to a constant only after a long time, the value of $I$ (or $J$ ) is very large. To overcome the drawback, we may first obtain a number of large values of $\Delta t$ such that the values of $I$ and $J$ are not too large, and then find the stationary probabilities for each value of $\Delta t$. The stationary probabilities when $\Delta t \rightarrow 0$ may next be obtained by means of extrapolation on the values of $\Delta t$. 
TABLE 8: Comparison of stationary sojourn time distributions computed by using the proposed numerical method and that given in Kim and Chaudhry [11] $(\Delta t=1.0)$.

\begin{tabular}{|c|c|c|c|c|}
\hline \multirow{3}{*}{ Time, $t$} & \multicolumn{2}{|c|}{ Example 1} & \multicolumn{2}{|c|}{ Example 2} \\
\hline & \multicolumn{2}{|c|}{$\begin{array}{c}\text { Sojourn Time Distribution } \\
{\left[T(z)=z / 10+3 z^{2} / 10+2 z^{3} / 5+z^{4} / 5, S(z)=3 z / 10+3 z^{2} / 5+z^{3} / 10\right]}\end{array}$} & \multicolumn{2}{|c|}{$\begin{array}{c}\text { Sojourn Time Distribution } \\
{\left[T(z)=z(z / 2+1 / 2)^{38}, S(z)=\left(z+z^{2}+\cdots+z^{35}\right) / 35\right]}\end{array}$} \\
\hline & Numerical method & Kim and Chaudhry [11] & Numerical method & Kim and Chaudhry [11] \\
\hline 0 & 0 & 0 & 0 & 0 \\
\hline 1 & 0.254329 & 0.254329 & 0.006915 & 0.006915 \\
\hline 2 & 0.543725 & 0.543708 & 0.007410 & 0.007408 \\
\hline 3 & 0.163404 & 0.163375 & 0.007911 & 0.007913 \\
\hline 4 & 0.030347 & 0.030357 & 0.008432 & 0.008429 \\
\hline 5 & 0.006524 & 0.006540 & 0.008968 & 0.008956 \\
\hline 6 & 0.001326 & 0.001339 & 0.009503 & 0.009493 \\
\hline 7 & $2.74 E-04$ & 0.000279 & 0.010059 & 0.010039 \\
\hline 8 & $5.66 E-05$ & 0.000058 & 0.010610 & 0.010595 \\
\hline 9 & $1.16 E-05$ & 0.000012 & 0.011178 & 0.011160 \\
\hline 10 & $2.40 E-06$ & 0.000002 & 0.011746 & 0.011731 \\
\hline$\vdots$ & $\vdots$ & $\vdots$ & $\vdots$ & $\vdots$ \\
\hline
\end{tabular}

TABLE 9: Comparison of stationary sojourn time distributions computed using the proposed numerical method and that given in Kim and Chaudhry [11] $(\Delta t=1.0)$.

\begin{tabular}{|c|c|c|c|c|}
\hline \multirow{3}{*}{ Time, $t$} & \multicolumn{2}{|c|}{ Example 3} & \multicolumn{2}{|c|}{ Example 4} \\
\hline & \multicolumn{2}{|c|}{$\begin{array}{r}\text { Sojourn Time Distribution } \\
{\left[T(z)=z(z / 2+1 / 2)^{38}, S(z)=\left(z+z^{2}+z^{3}\right) / 3\right]}\end{array}$} & \multicolumn{2}{|c|}{$\begin{array}{c}\text { Sojourn Time Distribution } \\
{\left[T(z)=z(z / 2+1 / 2)^{38}, S(z)=\left(z+z^{2}+\cdots+z^{19}\right) / 19\right]}\end{array}$} \\
\hline & Numerical method & Kim and Chaudhry [11] & Numerical method & Kim and Chaudhry [11] \\
\hline 0 & 0 & 0 & 0 & 0 \\
\hline 1 & 0.333333 & 0.333333 & 0.050290 & 0.050290 \\
\hline 2 & 0.333333 & 0.333333 & 0.051192 & 0.051193 \\
\hline 3 & 0.333333 & 0.333333 & 0.051803 & 0.051804 \\
\hline 4 & $1.62 E-11$ & $1.62 E-11$ & 0.052186 & 0.052187 \\
\hline 5 & $3.97 E-13$ & $4.04 E-13$ & 0.052408 & 0.052408 \\
\hline 6 & $\vdots$ & $\vdots$ & $\vdots$ & $\vdots$ \\
\hline
\end{tabular}

The method proposed in this paper may also be applied to other queuing models which cannot be represented as continuous-time Markov chains.

\section{Conflict of Interests}

The authors declare that there is no conflict of interests regarding the publication of this paper.

\section{References}

[1] M. H. Ackroyd, "Computing the waiting time distribution for the $G / G / 1$ queue by signal processing methods," IEEE Transactions on Communications, vol. 28, no. 1, pp. 52-58, 1980.
[2] M. J. Fryer and C. B. Winsten, "An algorithm to compute the equilibrium distribution of a one-dimensional bounded random walk," Operations Research, vol. 34, no. 3, pp. 449-454, 1986.

[3] W. K. Grassmann and J. L. Jain, "Numerical solutions of the waiting time distribution and idle time distribution of the arithmetic GI/G/1 queue," Operations Research, vol. 37, no. 1, pp. 141$150,1989$.

[4] G. Haßlinger, "Waiting time, busy periods and output models of a server analyzed via Wiener-Hopf factorization," Performance Evaluation, vol. 40, no. 1-3, pp. 3-26, 2000.

[5] J. Ponstein, "Theory and solution of a discrete queueing problem," Statistica Neerlandica, vol. 20, pp. 139-152, 1974.

[6] A. G. Konheim, "An elementary solution of the queuing system G/G/1," SIAM Journal on Computing, vol. 4, no. 4, pp. 540-545, 1975. 
[7] G. Haßlinger, "A polynomial factorization approach to the discrete time GI/G/1/(N) queue size distribution," Performance Evaluation, vol. 23, no. 3, pp. 217-240, 1995.

[8] A. S. Alfa and W. Li, "Matrix-geometric analysis of the discrete time GI/G/1 system," Stochastic Models, vol. 17, no. 4, pp. 541$554,2001$.

[9] A. S. Alfa, "Combined elapsed time and matrix-analytic method for the discrete time $G I / G / 1$ and $G I^{x} / G / 1$ systems," Queueing Systems: Theory and Applications, vol. 45, no. 1, pp. 5-25, 2003.

[10] B. V. Rao and R. M. Feldman, "Numerical approximations for the steady-state waiting times in a GI/G/1 queue," Queueing Systems, vol. 31, no. 1-2, pp. 25-42, 1999.

[11] N. K. Kim and M. L. Chaudhry, "The use of the distributional Little's law in the computational analysis of discrete-time GI/G/1 and GI/D/c queues," Performance Evaluation, vol. 65, no. 1, pp. 3-9, 2008.

[12] M. F. Neuts, Matrix-Geometric Solutions in Stochastic Models: An Algorithmic Approach, The Johns Hopkins University Press, Baltimore, Md, USA, 1981.

[13] W. S. Yang, D. E. Lim, and K. C. Chae, "Maintenance of deteriorating single server queues with random shocks," Computers and Industrial Engineering, vol. 57, no. 4, pp. 1404-1406, 2009.

[14] S. K. Koh, Maintenance of deteriorating non-exponential single server queue [Ph.D. thesis], Institute of Mathematical Sciences, University of Malaya, Kuala Lumpur, Malaysia, 2013.

[15] D. Gross and C. M. Harris, Fundamentals of Queueing Theory, John Wiley \& Sons, New York, NY, USA, 1998. 


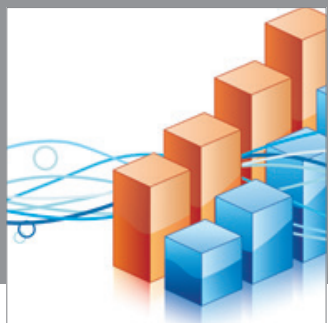

Advances in

Operations Research

mansans

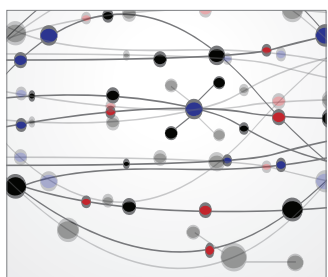

The Scientific World Journal
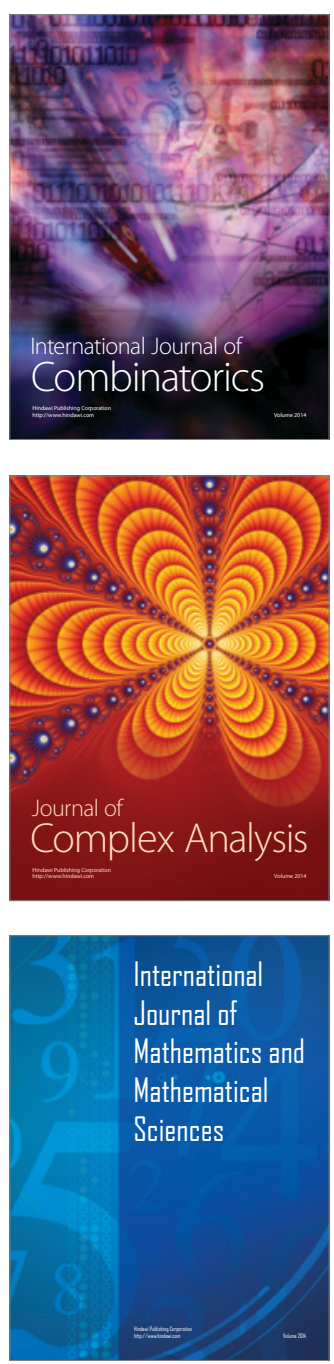
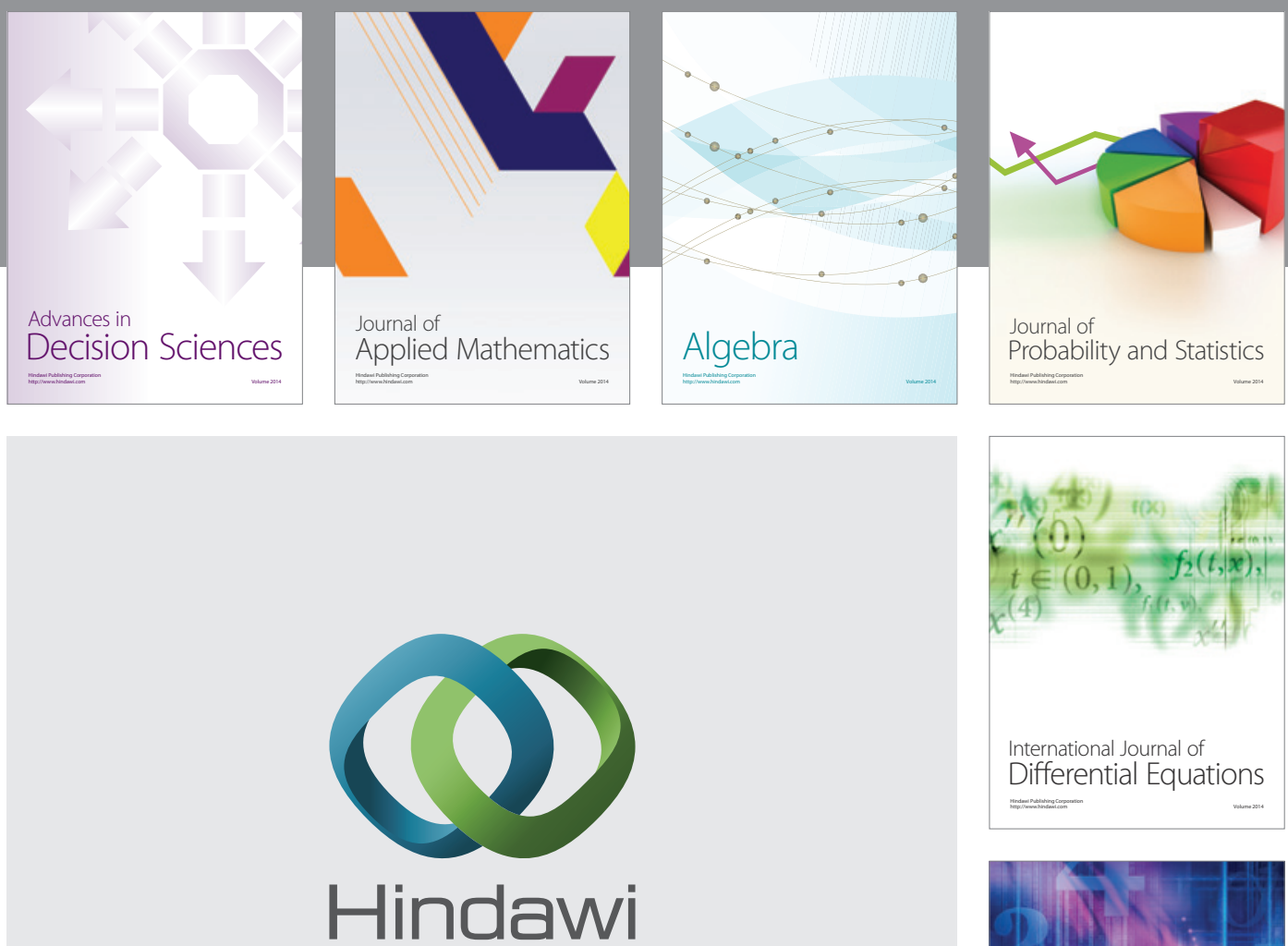

Submit your manuscripts at http://www.hindawi.com
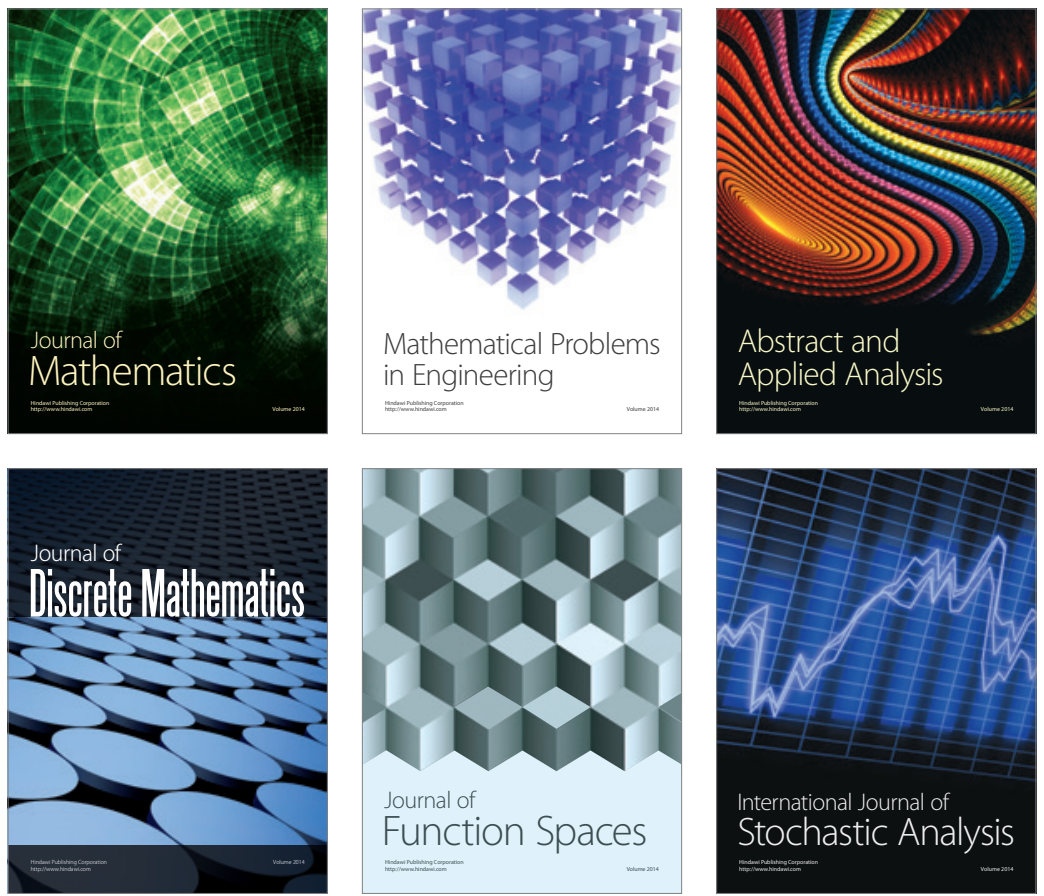

Journal of

Function Spaces

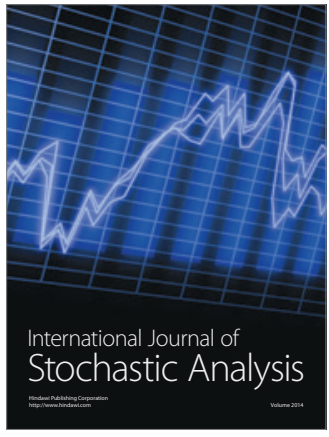

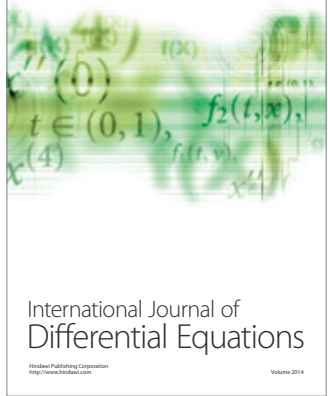
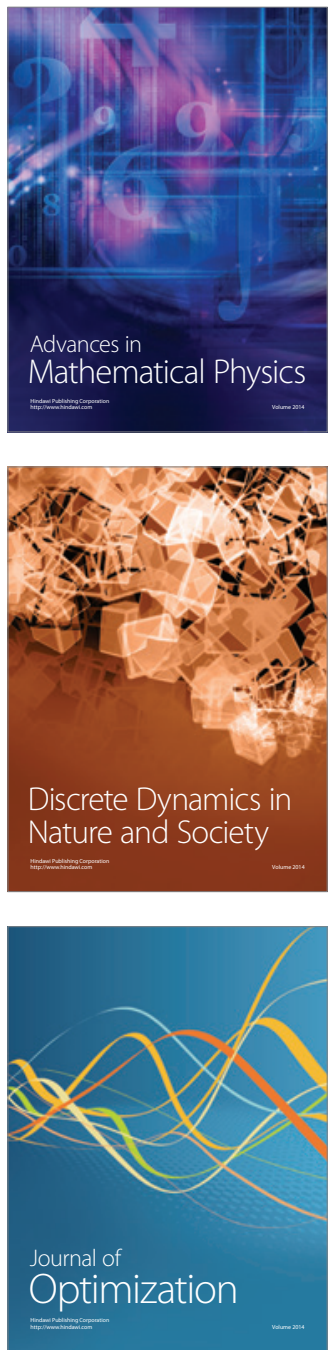